\title{
Guest Editorial: Special Issue on Control of Quantum Mechanical Systems
}

I $\mathrm{T}$ is perhaps not too surprising (at least for a control audience) that "manipulating and controlling things at a small scale" was already a key part of the program that Richard P. Feynman formulated in his 1959 visionary lecture [1] which is commonly recognized as the birth place of Quantum Information. Quoting from [1]: "The principles of Physics as far as I can see do not speak against the possibility of maneuvering things atom by atom. It is not an attempt to violate any laws; it is something, in principle, that can be done; but in practice, it has not been done because we are too big". Fifty years later, if we are "small enough" to store the "Encyclopædia Britannica on the head of a pin," we are still "too big" to carry out Feynman program at the atomic scale, although we are getting closer. Getting closer, however, requires passing from nanotechnology to manipulating and controlling quantum systems, and this is no longer a question of scales, as quantum mechanics obeys its own laws. For example, observing a quantum system disturbs its state, which complicates a number of "standard" control tasks, most notably measurement-based feedback.

The enormous technological progress in all the experimental fields in which control at quantum level is of interest (ranging from physical chemistry to quantum optics, from spectroscopy to nanomechanics and superconducting quantum circuits) are motivating the development of new analytic and computational tools. If quantum physics has been for a century a descriptive science, we are now assisting to a paradigmatic shift towards quantum engineering. In this context, systematic control design methods (of both theoretical and practical nature), tailored to the quantum mechanical setting, are strongly needed and will be even more in the near future.

The rationale behind this Special Issue is therefore to present an overview of the state of the art of the field and to stimulate researchers in control theory to get involved, by gathering together samples of control-oriented research currently going on. While dedicated special issues have appeared in recent years in various physics journals (see for example New Journal of Physics, vol. 11, October 2009, or Journal of Physics B: At. Molecular and Optical Physics, vol. 44, August 2011), this is the first time a thematic issue on these topics appears on a major control journal.

Of the 29 submissions we received in response to the call for papers, 14 appear in this Special Issue. They cover various aspects of quantum control, spanning from controllability

Digital Object Identifier 10.1109/TAC.2012.2202053 to optimal control, from open-loop control methods to feedback, in the different-and subtle-incarnations that this concept assumes passing from the classical to the quantum world. The tools used for the investigation are also widely different: from geometric to stochastic methods, passing through linear and nonlinear system-theoretic tools many of which should be familiar also to control scientists with a limited background in quantum physics.

To conclude this guest editorial, we would like to thank the IEEE TAC Editorial Board, its past and current Editor-in-Chief, Christos Cassandras and Panos Antsaklis who were supportive of this Special Issue from day one to its completion, and Editorial Assistant Elizabeth Kováks for her constant assistance in editorial matters. Finally, and most importantly, we would like to express our gratitude to all authors who contributed with their submissions and to the reviewers who took care of screening those papers in a very professional way.

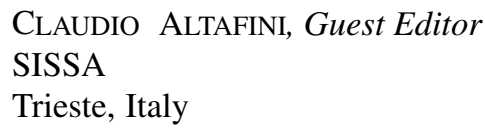

ANTHONY M. BLOCH, Guest Editor Univ. of Michigan

Ann Arbor, MI

MATTHEW R. JAMES, Associate Editor Australian National University Canberra, Australia

ANTONIO LORIA, Associate Editor CNRS

Gif-sur-Yvette, France

PIERRE RoUCHON, Guest Editor

MinesParisTech

Paris, France

\section{REFERENCES}

[1] R. P. Feynman, "There is plenty of room at the bottom. An invitation to enter a new field of Physics," Transl.:Transcript of the talk given at the 1959 Annual Meeting of the American Physical Society. Caltech Eng. Sci., vol. 23, no. 5, pp. 22-36, 1960. 

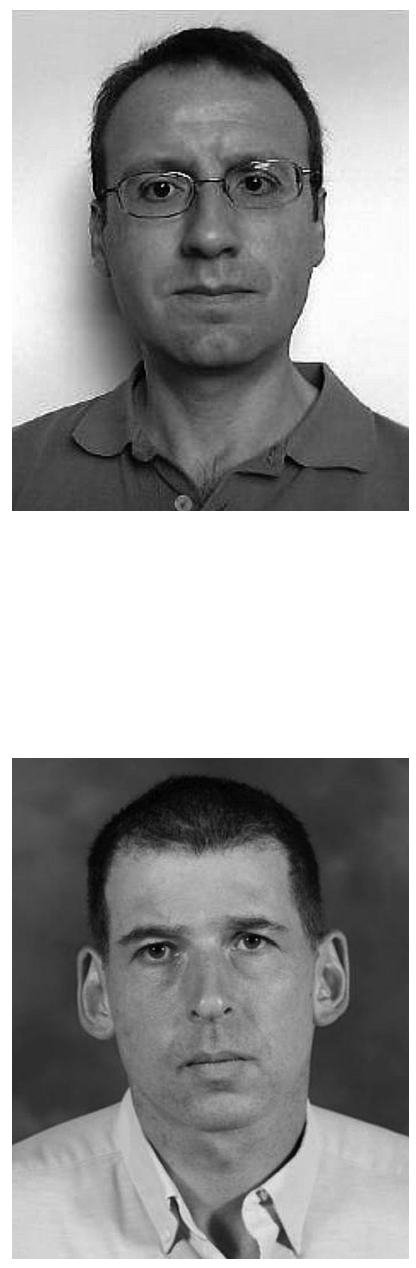

Anthony M. Block (S'84-M'85-SM'02-F'03) received the B.Sc. degree (with honors) from the University of the Witwatersrand, Johannesburg, South Africa, in 1978, the M.S. degree from the California Institute of Technology, Pasadena, in 1979, the M.Phil degree from Cambridge University, Cambridge, U.K., in 1981, and the Ph.D. degree from Harvard University, Cambridge, MA, in 1985 .

He is the Alexander Ziwet Collegiate Professor of Mathematics at the University of Michigan, Ann Arbor. He is an author of Nonholomic Mechanics and Control (Springer-Verlag, 2003). His research interests are in mechanics and nonlinear control, Hamiltonian systems and integrable systems, and related areas of nonlinear dynamics.

Dr. Bloch received a Presidential Young Investigator Award and a Guggenheim Fellowship and has been a member of IAS. He was Associate Editor at Large for the IEEE TRANSACTIONS ON AutOMATIC CONTROL, and is currently an Editor for several journals including Mathematics of Controls, Signals and Systems, the Journal of Nonlinear Science, and Systems and Controls Letters.

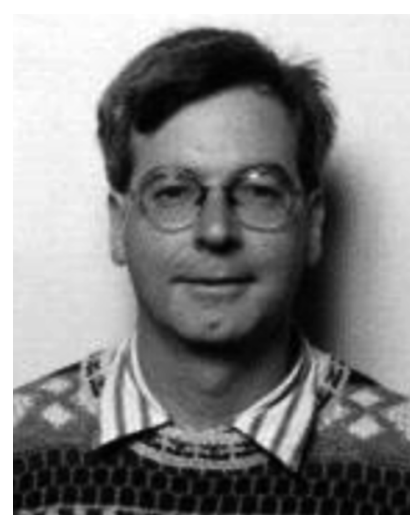

Matthew R. James (S'86-M'86-SM'00-F'02) was born in Sydney, Australia, in 1960. He received the B.Sc. degree in mathematics and the B.E. degree (Hon. I) in electrical engineering from the University of New South Wales, Sydney, Australia, in 1981 and 1983, respectively, and the Ph.D. degree in applied mathematics from the University of Maryland, College Park, in 1988.

In 1988 and 1989, he was a Visiting Assistant Professor with the Division of Applied Mathematics, Brown University, Providence, RI, and from 1989 to 1991 he was an Assistant Professor with the Department of Mathematics, University of Kentucky, Lexington. In 1991, he joined the Australian National University, Australia, where he served as Head of the Department of Engineering during 2001 and 2002. He has held visiting positions with the University of California, San Diego, Imperial College, London, and the University of Cambridge. His research interests include quantum, nonlinear, and stochastic control systems.

Dr James received the SIAM Journal on Control and Optimization Best Paper Prize for 2007. He is currently serving as Associate Editor for the IEEE TRANSACTIONS ON AUTOMATIC CONTROL, and has previously served the SIAM Journal on Control and Optimization, Automatica, and Mathematics of Control, Signals, and Systems. He has held an Australian Research Council Professorial Fellowship. 

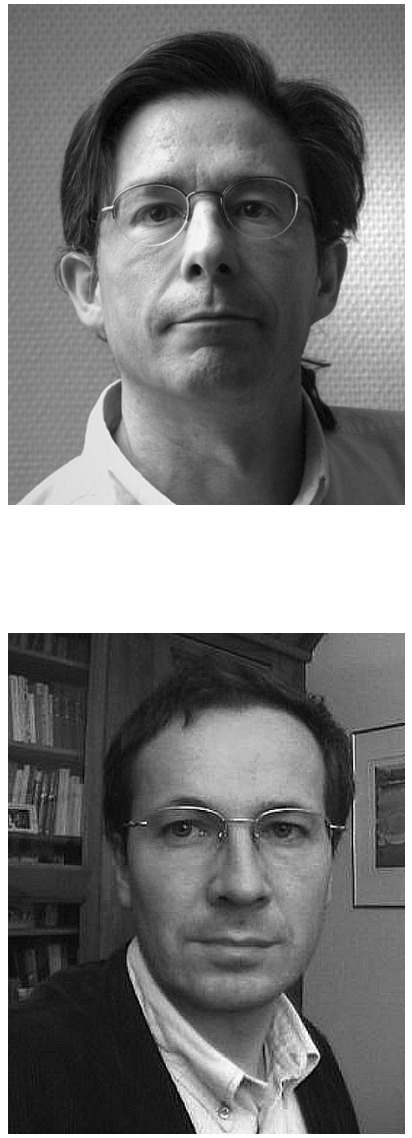

Antonio Loria (M'91) was born in Mexico City, Mexico, in 1969. He received the B.Sc. degree in electronic engineering from the ITESM, Monterrey, Mexico, in 1991, and the M.Sc. and Ph.D. degrees in control engineering from the Université de Technologie de Compiègne (UTC), Compiègne, France, in 1993 and 1996, respectively.

From 1996 to 1998, he was successively an Associate Researcher at the University of Twente, The Netherlands; NTNU, Norway, and the CCEC, University of California at Santa Barbara. He has the honour of holding a research position as "Directeur de Recherche" (senior researcher), at the French National Centre of Scientific Research (CNRS). His research interests include control systems theory and practice, electrical systems, analysis and control of chaos.

Dr. Loria is an Associate Editor for Systems and Control Letters, Automatica, the IEEE TRANSACtions on Automatic Control, the IEEE Transactions on Control Systems TECHNOLOGY, and is member of the IEEE CSS Conference Editorial Board.

Pierre Rouchon was born in Saint-Etienne, France, in 1960. He received the M.S. degree from Ecole Polytechnique, Palaiseau, France, in 1983, the Ph.D. degree in chemical engineering from Mines ParisTech, Paris, France, in 1990, and the "habilitation diriger des recherches" degree in mathematics from the University Paris-Sud, Orsay, in 2000.

From 1993 to 2005, he was an Associate Professor in applied mathematics at Ecole Polytechnique. From 1998 to 2002, he was the head of the Centre Automatique et SystËmes, Mines ParisTech. He is now a Professor at Mines ParisTech. His fields of interest include nonlinear control, system theory and its applications. His contributions include differential flatness and its extension to infinite dimensional systems, nonlinear observers and symmetries, process control, motion planing and tracking for mechanical systems, feedback stabilization and estimation for electrical drives, internal combustion engines and quantum systems. 